\title{
In vitro uptake of recombinant factor VIla by megakaryocytes with subsequent production of platelets containing functionally active drug
}

Recombinant activated factor VII (rFVIIa) has been recently shown to prevent spontaneous bleeding in inhibitorcomplicated haemophilia when administered once daily (Konkle et al, 2007; Young et al, 2012). The pro-haemostatic effect of rFVIIa prophylaxis is difficult to explain given its plasma half-life of $2 \mathrm{~h}$.

In the literature, four mechanisms explaining the prophylactic efficacy of once-daily rFVIIa administration have been proposed, including the requirement of much lower doses of rFVIIa to prevent than to treat bleeding, improvement of endothelial cell permeability, uptake of rFVIIa by platelets, and redistribution of rFVIIa into the extravascular space (Lisman \& de Groot, 2015). Accumulation of rFVIIa in bone and joints may explain the prophylactic activity of rFVIIa. One study suggested that rFVIIa was taken up by megakaryocytes within the bone marrow (Gopalakrishnan et al, 2010).

The present study tested the hypothesis that megakaryocytes that have taken up rFVIIa will produce rFVIIa-containing platelets, which may explain the prolonged haemostatic effect of rFVIIa in a prophylactic setting.
We used the megakaryoblastic cell line MEG-01, which was cultured and differentiated by valproic acid as described earlier (Kirschbaum et al, 2015). After at least $10 \mathrm{~d}$ of differentiation, different concentrations of rFVIIa were added to the culture medium and after $2 \mathrm{~h}$, MEG-01 cells were either harvested or stimulated to produce platelet-like particles (PLPs) by addition of $100 \mathrm{ng} / \mathrm{ml}$ recombinant human thrombopoietin (rTHPO; Life Technologies, Carlsbad, CA, USA) for $3 \mathrm{~d}$. Cells or PLPs were harvested by centrifugation, washed and lysed by freeze-thawing the samples twice.

We first showed that rFVIIa was dose-dependently taken up by MEG-01 cells (Fig 1A). After 3 d of rTHPO stimulation, hardly any rFVIIa was detected in the MEG-01 cells (Fig 1B). In contrast, the PLPs produced from these MEG-01 cells contained appreciable amounts of rFVIIa (Fig 1C), which suggests rFVIIa is selectively transferred to PLPs. Active selection of agents to be transferred to platelets in the process of megakaryocyte maturation has been previously demonstrated, as megakaryocytes transfer some, but not all, mRNA species examined to platelets (Cecchetti et al, 2011).

Fig 1. Dose-dependent uptake of rFVIIa by MEG-01 cells with subsequent production of PLPs containing functionally active rFVIIa. (A-C) rFVIIa was added in different concentrations $(0-100 \mathrm{nmol} / \mathrm{l})$ to valproic acid-stimulated MEG-01 cells, which were harvested after $2 \mathrm{~h}$ (A), or stimulated with $100 \mathrm{ng} / \mathrm{ml}$ recombinant human thrombopoietin to produce platelet-like particles (PLPs). After 3 d, MEG-01 cells (B) or PLPs (C) were isolated by centrifugation. Lysates of MEG-01 cells and PLPs were tested for rFVIIa content using a microtitre plate-based clotting assay in factor VII-depleted plasma as previously described (Schut et al, 2014), and rFVIIa levels in the lysates were expressed relative to the total protein content of these lysates (Pierce BCA protein assay kit, Thermo Scientific, Hanover Park, IL, USA). A calibration curve of rFVIIa was used to convert clotting times to rFVIIa concentrations. Shown are means of 3 independent experiments, error bars indicate standard error of mean (SEM). (D) Immunofluorescent stains of MEG-01 cells (i-iii) or PLPs (iv) using an antibody to factor VII(a). Shown are typical examples of MEG-1 cells that were exposed to $100 \mathrm{nmol} / \mathrm{l} \mathrm{rFVIIa}$ for $2 \mathrm{~h}$ (i) or $3 \mathrm{~d}$ (iii) or cells that were exposed for $2 \mathrm{~h}$ to vehicle (ii). In addition, PLPs derived from MEG-01 cells exposed to $100 \mathrm{nmol} / \mathrm{l}$ of rFVIIa harvested at day 3 are shown (iv). Cells were permeabilized with $0 \cdot 1 \%$ (v/v) triton X-100 in phosphate-buffered saline (PBS) for $10 \mathrm{~min}$ at room temperature, and stained with $5 \mu \mathrm{g} / \mathrm{ml}$ monoclonal mouse anti-human FVII followed by $5 \mu \mathrm{g} / \mathrm{ml}$ Alexa Fluor 488 goat anti-mouse antibody and counterstained with 4 units/ml Alexa Fluor 594 phalloidin (A12381; Life Technologies, Carlsbad, CA, USA). This staining was used to indicate the cell membrane, but for clarity the staining itself is not shown in the figures. All samples were fixed using Vectashield Hardset mounting medium with DAPI (Vector Laboratories Inc. Burlingame, CA, USA). rFVIIa is stained in green, nuclei in blue, and the cell membrane is represented by the dotted line. Scale bars represent $20 \mu \mathrm{m}$ (MEG-01 cells) or $10 \mu \mathrm{m}$ (PLPs). Original magnification $1000 \times$. (E) Flow cytometry analysis of PLPs derived from MEG-01 cells that have been exposed to $100 \mathrm{nmol} / \mathrm{l}$ of rFVIIa using permeabilized (green line) or non-permeabilized (blue line) cells. The black line represents PLPs generated from MEG-01 cells that not have been exposed to rFVIIa. PLPs were fixed with $2 \%(\mathrm{v} / \mathrm{v})$ formaldehyde in $0.9 \% \mathrm{NaCl}$ for 10 min at room temperature and permeabilized with $0 \cdot 1 \%(\mathrm{v} / \mathrm{v})$ triton X-100 in PBS or vehicle for $10 \mathrm{~min}$ at room temperature. After washing, samples were stained for $\mathrm{rFVIIa} u s i n g 10 \mu \mathrm{g} / \mathrm{ml}$ monoclonal mouse anti-human FVII [ABIN951602, clone AA-3, antibodies-online, Germany (www.antikoerper-online.de)] for $1 \mathrm{~h}$ at room temperature followed by $10 \mu \mathrm{g} / \mathrm{ml}$ Alexa Fluor 488-labelled goat anti-mouse antibody (A-11001; Life Technologies) for 30 min at room temperature. Data shown is representative of three independent experiments. (F, G) PLPs derived from MEG-01 cells exposed to $100 \mathrm{nmol} / \mathrm{l} \mathrm{rFVIIa}$ or vehicle were added to normal pool plasma (NPP), factor VII or factor VIII depleted plasma at a final concentration of 10000 PLPs/ $\mu$ l plasma, and tested by calibrated automated thrombography using the platelet-rich plasma (PRP) reagent (Thrombinoscope BV, Maastricht, The Netherlands). Shown are lag time (F) values derived from thrombin generation curves and representative thrombin generation curves (G). Data represent the mean of three independent experiments. Error bars indicate standard deviation. ${ }^{*} P<0.05,{ }^{*} P<0.01, * * * P<0.001$ by paired $t$-test. def., deficient. 


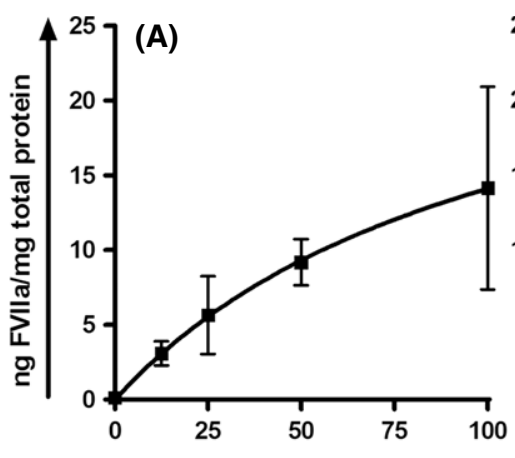

(B)

(D)

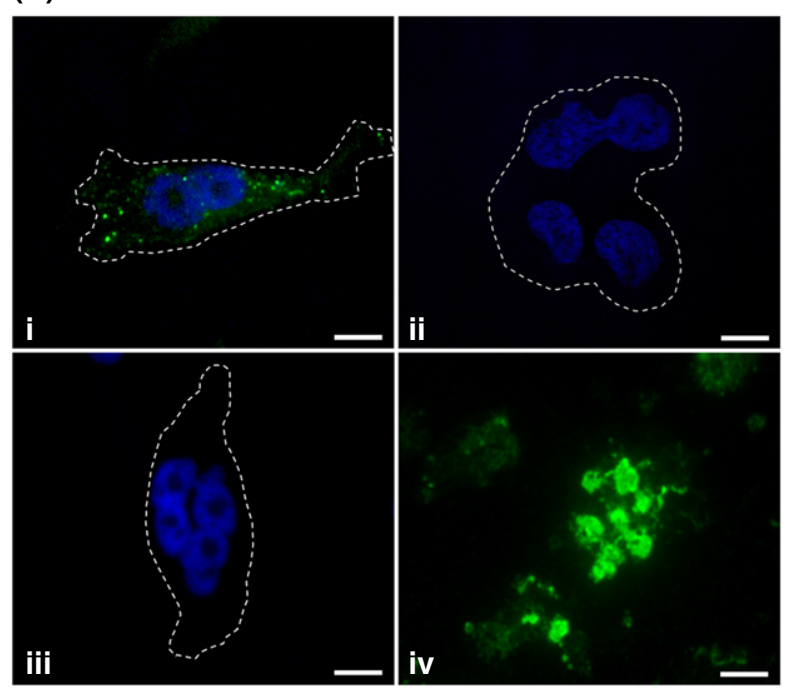

(E)

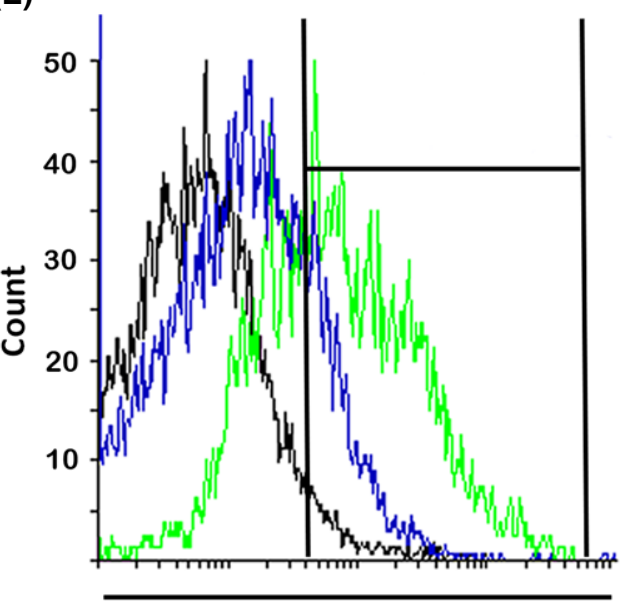

rFVIIa-Alexa 488

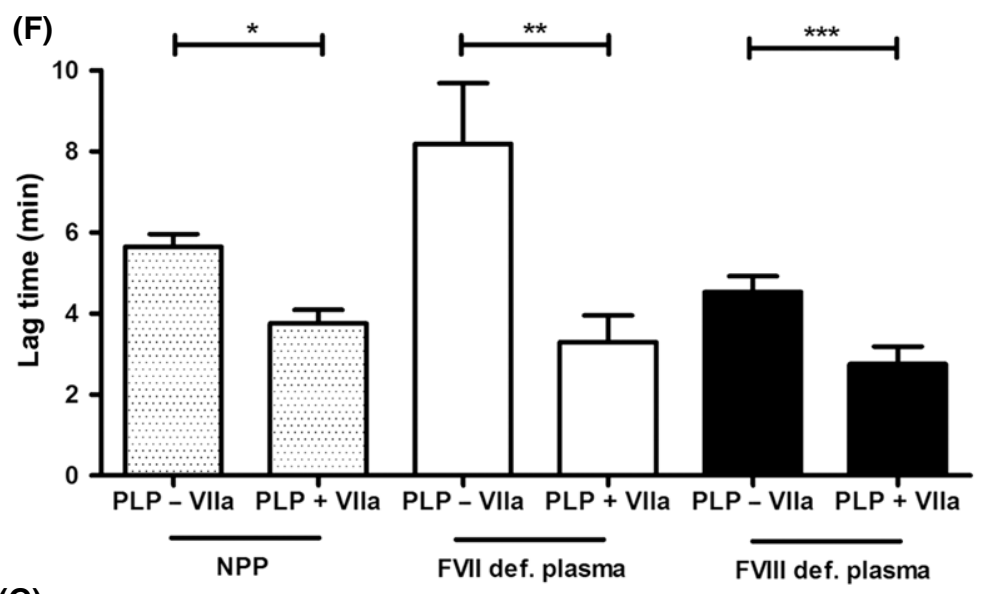

(G)

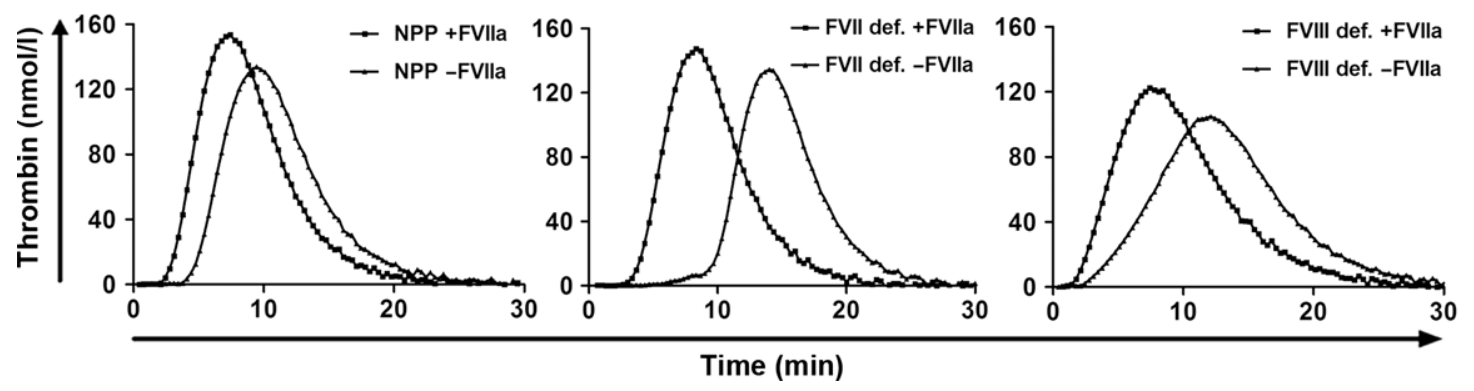


(A)

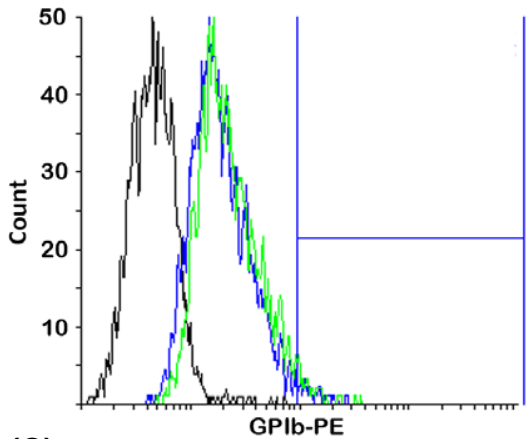

(C)
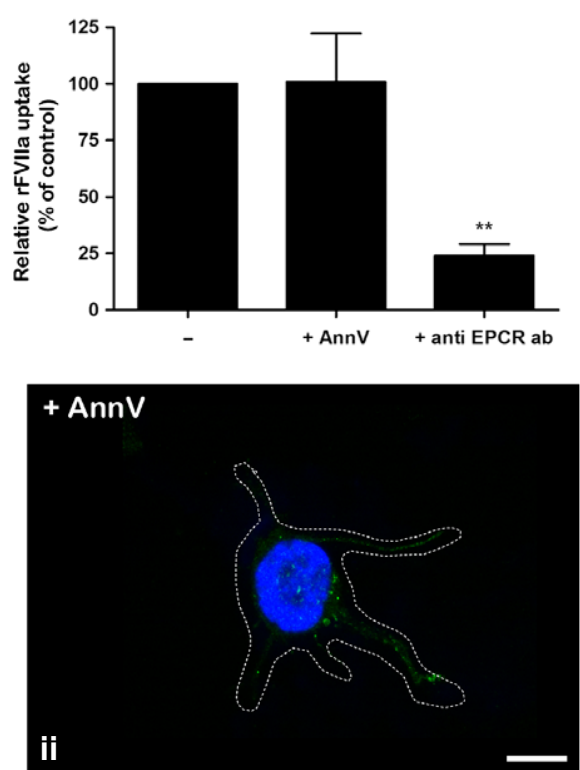

(B)

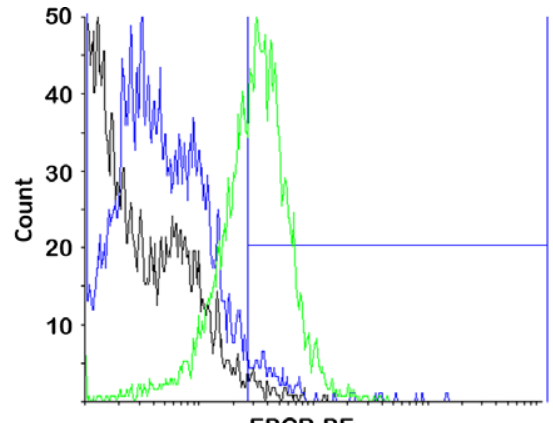

(D)
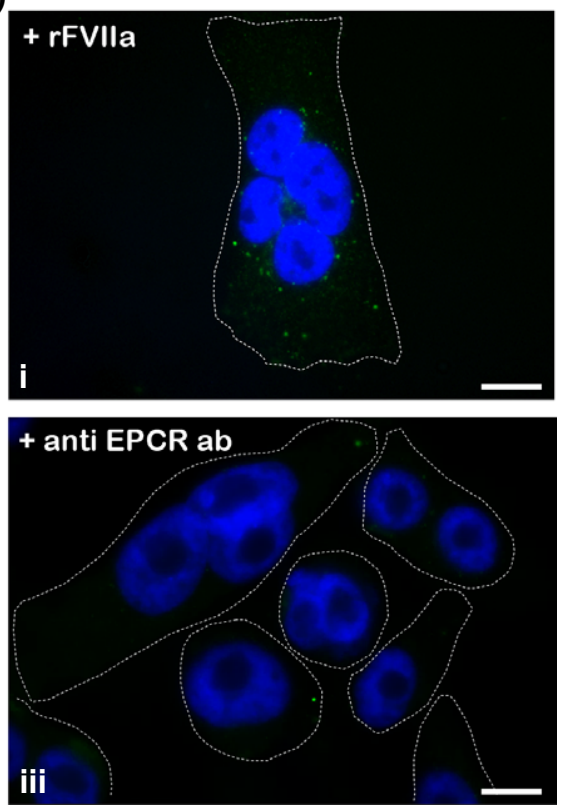

Fig 2. rFVIIa uptake by MEG-01 cells is mediated by endothelial protein C receptor (EPCR). (A, B) MEG-01 cells were stained for glycoprotein Ib $\alpha$ (A) using $156 \mu \mathrm{g} / \mathrm{ml} \mathrm{R-Phycoerythrin} \mathrm{(PE)-labelled} \mathrm{monoclonal} \mathrm{mouse} \mathrm{anti-human} \mathrm{CD42b} \mathrm{(GPIb,} \mathrm{clone} \mathrm{AN51,} \mathrm{Dako} \mathrm{Denmark,} \mathrm{Glostrup,} \mathrm{Den-}$ mark) or EPCR (B) using $10 \mu \mathrm{g} / \mathrm{ml}$ R-PE-labelled rat anti-human CD201 antibody (EPCR, clone RCR-401, Biolegend, San diego, CA, USA) for $20 \mathrm{~min}$ at $37^{\circ} \mathrm{C}$ and analysed by flow cytometry. The black lines represent the background fluorescence of the MEG-01 cells, and the blue lines correspond to appropriate isotype controls. Data shown is representative of three independent experiments. (C) MEG-01 cells were incubated with $100 \mathrm{nmol} / \mathrm{l}$ of rFVIIa for $2 \mathrm{~h}$ in presence or absence of $30 \mu \mathrm{g} / \mathrm{ml}$ Annexin V (AnnV) or $45 \mu \mathrm{g} / \mathrm{ml} \mathrm{rat} \mathrm{monoclonal} \mathrm{anti-EPCR} \mathrm{antibody} \mathrm{(RCR-252;}$ Novus Biologicals, Littleton, CO, USA), and rFVIIa content of cell lysates was determined by a microtitre plate-based clotting assay using factor VIIdepleted plasma. Shown is the quantity of rFVIIa in cell lysates expressed as percentage of control. Data represents the mean with error bars indicating SEM $(n=3)$. ${ }^{* * P} \leq 0 \cdot 01$, compared to control (-) by one-way analysis of variance with Dunnett's post-test. (D) Immunofluorescent staining of MEG-01 cells under the experimental conditions described in panel C. Staining details are given in the legend of Fig 1D. rFVIIa is stained in green, nuclei in blue, and the cell membrane is represented by the dotted line. Scale bars indicate $20 \mu \mathrm{m}$. Original magnification $630 \times$. ab, antibody.

To confirm that rFVIIa is taken up by MEG-01 cells (and not just associated with the cell), we stained MEG-01 cells or PLPs for rFVIIa and examined cells using fluorescence microscopy. There was an ubiquitous presence of rFVIIa within the vast majority of cells (80-90\%) in an apparent punctuate pattern at $2 \mathrm{~h}$ after the addition of rFVIIa to the culture medium (Fig 1D i), whereas no staining was detected in cells that had not been exposed to rFVIIa (Fig 1D ii). After $3 \mathrm{~d}$ of rTHPO stimulation, no rFVIIa staining was detected in the MEG-01 cells (Fig 1D iii). The MEG-01 cellderived PLPs harvested at day 3, however, did stain positive for rFVIIa (Fig 1D iv). We also used flow cytometry to confirm the presence of rFVIIa within PLPs. rFVIIa was predominantly localized within the PLP because 55.6\% $\pm 11 \cdot 3 \%$ [mean $\pm \mathrm{SD}, n=3]$ of permeabilized cells stained positive for rFVIIa, compared to $19 \cdot 9 \% \pm 5 \cdot 1 \%$ [mean $\pm \mathrm{SD}, n=3$ ] of non-permeabilized cells (Fig 1E).

rFVIIa within PLPs is haemostatically active, as levels were determined in PLP lysates with a functional coagulation assay. To determine whether this functionally active rFVIIa contributes to haemostasis in a plasma environment, we performed in vitro thrombin generation measurements using plasma to which intact PLPs were added. Addition of rFVIIa-containing PLPs resulted in a profound shortening of 
the lag time of the thrombin generation curve in normal, FVII-, and FVIII-depleted plasma, compared to addition of PLPs generated from MEG-01 cells that had not been exposed to rFVIIa (Fig 1F). rFVIIa-containing PLPs also had a slight but significantly increased peak thrombin generation compared to control PLPs (Fig 1G). Whether rFVIIa in PLPs does not encounter inhibitors (such as tissue factor pathway inhibitor and antithrombin) or whether the rFVIIa measured in our assay only represents a fraction of total rFVIIa in PLPs, with the remainder being in complex with an inhibitor, requires further study.

We next assessed the mechanism of uptake of rFVIIa by MEG-01 cells. We hypothesized a role for negatively charged phospholipids, glycoprotein (GP)Ib $\alpha$ and endothelial protein $\mathrm{C}$ receptor (EPCR), which are all known binding partners for rFVIIa (Lisman \& de Groot, 2015). GPIb $\alpha$ was hardly detected on the surface of differentiated MEG-01 cells: $2 \cdot 5 \% \pm 0 \cdot 6 \%[($ mean $\pm \mathrm{SD}, n=3)$ of cells stained positive for GPIb $\alpha$, Fig 2A]. In contrast, EPCR was abundantly present on MEG-01 cells with $73 \cdot 2 \% \pm 11 \cdot 8 \%$ (mean \pm SD, $n=3$ ) of cells staining positive (Fig $2 \mathrm{~B}$ ), which has, to our knowledge, not been reported before. Uptake of rFVIIa was not affected by Annexin A5, excluding a role for negatively charged phospholipids in rFVIIa uptake, but a $76 \cdot 0 \% \pm 8 \cdot 7 \%$ [mean $\pm \mathrm{SD}$ ] reduction of rFVIIa uptake was observed in the presence of an antibody to EPCR (Fig 2C). Immunofluorescent staining of rFVIIa confirmed these results (Fig 2D). EPCR appears to fulfil multiple critical features in the mode of action of rFVIIa, including enhancement of haemostatic activity in the intravascular space (Pavani et al, 2014), improvement of endothelial barrier function (Sundaram et al, 2014), transport of rFVIIa to extravascular sites (Clark et al, 2012) and uptake by megakaryocytes in the bone marrow (this study).

Taken together, we demonstrate EPCR-dependent uptake of rFVIIa by megakaryocytes with subsequent production of rFVIIa-containing 'prohaemostatic' platelets. Whether this mechanism acts in vivo requires further study, but delayed generation of rFVIIa-containing platelets appears a plausible mechanism to partly explain the efficacy of once-daily rFVIIa prophylaxis in inhibitor-complicated haemophilia.

\section{Author contributions}

A.M. Schut and M. Kirschbaum participated in the design of the study, performed experiments, analysed and interpreted data and wrote the manuscript. J. Adelmeijer performed experiments, analysed and interpreted data. P.G. de Groot interpreted data. T. Lisman participated in the design of and supervised the study, interpreted data and wrote the manuscript. All authors revised and approved the manuscript.

\section{Financial support}

This study was supported in part by an unrestricted educational grant from Novo Nordisk and by a grant from the Netherlands Organisation of Scientific Research (VIDI, 917.11.304 to T.L.).

\section{Conflict of interest}

This study was supported by an unrestricted educational grant from Novo Nordisk, whose product is studied in the manuscript.

\author{
Anne Marieke Schut ${ }^{1}$ \\ Marc Kirschbaum ${ }^{1}$ \\ Jelle Adelmeijer ${ }^{1}$ \\ Philip G. de Groot ${ }^{2}$ \\ Ton Lisman ${ }^{1}$ \\ ${ }^{1}$ Surgical Research Laboratory, Department of Surgery, University of \\ Groningen, University Medical Centre Groningen, Groningen, and \\ ${ }^{2}$ Department of Clinical Chemistry and Haematology, University of \\ Utrecht, University Medical Centre Utrecht, Utrecht, The Netherlands. \\ E-mail: j.a.lisman@umcg.nl
}

Keywords: haemophilia, megakaryocytes, blood platelets, recombinant FVIIa, prophylaxis

First published online 12 June 2016

doi: $10.1111 /$ bjh.14149

\section{References}

Cecchetti, L., Tolley, N.D., Michetti, N., Bury, L., Weyrich, A.S. \& Gresele, P. (2011) Megakaryocytes differentially sort mRNAs for matrix metalloproteinases and their inhibitors into platelets: a mechanism for regulating synthetic events. Blood, 118, 1903-1911.

Clark, C.A., Vatsyayan, R., Hedner, U., Esmon, C.T., Pendurthi, U.R. \& Rao, L.V. (2012) Endothelial cell protein $\mathrm{C}$ receptor-mediated redistribution and tissue-level accumulation of factor VIIa. Journal of Thrombosis and Haemostasis, 10, 2383-2391.
Gopalakrishnan, R., Hedner, U., Ghosh, S., Nayak, R.C., Allen, T.C., Pendurthi, U.R. \& Rao, L.V. (2010) Bio-distribution of pharmacologically administered recombinant factor VIIa (rFVIIa). Journal of Thrombosis and Haemostasis, 8, 301310.

Kirschbaum, M., Karimian, G., Adelmeijer, J., Giepmans, B.N., Porte, R.J. \& Lisman, T. (2015) Horizontal RNA transfer mediates plateletinduced hepatocyte proliferation. Blood, 126, 798-806.

Konkle, B.A., Ebbesen, L.S., Erhardtsen, E., Bianco, R.P., Lissitchkov, T., Rusen, L. \& Serban, M.A. (2007) Randomized, prospective clinical trial of recombinant factor VIIa for secondary prophylaxis in hemophilia patients with inhibitors. Journal of Thrombosis and Haemostasis, 5, 19041913.

Lisman, T. \& de Groot, P.G. (2015) The role of cell surfaces and cellular receptors in the mode of action of recombinant factor VIIa. Blood Reviews, 29, 223-229.

Pavani, G., Ivanciu, L., Faella, A., MarcosContreras, O.A. \& Margaritis, P. (2014) The endothelial protein $\mathrm{C}$ receptor enhances hemostasis of FVIIa administration in hemophilic mice in vivo. Blood, 124, 11571165. 
Schut, A.M., Hyseni, A., Adelmeijer, J., Meijers, J.C., De Groot, P.G. \& Lisman, T. (2014) Sustained pro-haemostatic activity of rFVIIa in plasma and platelets in non-bleeding pigs may explain the efficacy of a once-daily prophylaxis in humans. Thrombosis and Haemostasis, 112, 304-310.
Sundaram, J., Keshava, S., Gopalakrishnan, R., Esmon, C.T., Pendurthi, U.R. \& Rao, L.V. (2014) Factor VIIa binding to endothelial cell protein C receptor protects vascular barrier integrity in vivo. Journal of Thrombosis and Haemostasis, 12, 690-700.
Young, G., Auerswald, G., Jimenez-Yuste, V., Lambert, T., Morfini, M., Santagostino, E. \& Blanchette, V. (2012) PRO-PACT: retrospective observational study on the prophylactic use of recombinant factor VIIa in hemophilia patients with inhibitors. Thrombosis Research, 130, 864-870.

\section{Autoimmune thrombotic thrombocytopenic purpura associated with HHV8-related Multicentric Castleman disease}

Thrombotic thrombocytopenic purpura (TTP) is a thrombotic microangiopathy (TMA) that results from severe ADAMTS13 (A Disintegrin And Metalloprotease with Thrombospondin type 1 motif member 13) deficiency. The main cause of TTP is autoantibodies against ADAMTS13 (Furlan et al, 1998; Tsai \& Lian, 1998).

Multicentric Castleman disease (MCD) is a rare lymphoproliferative disorder, marked by recurrent flares of fever, lymphadenopathy and splenomegaly. MCD presents with cytopenias and elevated $\mathrm{C}$ reactive-protein (CRP). MCD is more frequent in association with human immunodeficiency virus (HIV) infection. Human herpes virus 8 (HHV8) is responsible for the onset of MCD (HHV8/
MCD) in all HIV-associated MCD (HIV/MCD) and in half of HIV negative patients (Soulier et al, 1995; Oksenhendler et al, 1996).

Several autoimmune diseases have been associated with MCD (Muskardin et al, 2012). We report for the first time four cases of TTP associated with HHV8/MCD.

All the patients included in a prospective database for Castleman disease between 1 January 2003 and 1 October 2012 who presented with a TMA were included in this study. Patients' records were reviewed using a pre-established chart.

Thrombotic thrombocytopenic purpura was defined by TMA and severe ADAMTS13 deficiency $(<5 \%$ of normal activity). ADAMTS13 activity and anti-ADAMTS13

Table I. Characteristics of patients at TMA onset

\begin{tabular}{|c|c|c|c|c|c|}
\hline & Patient 1 & Patient 2 & Patient 3 & Patient 4 & Median \\
\hline Age (years) & 56 & 44 & 52 & 42 & 48 \\
\hline Sex & Male & Male & Male & Male & \\
\hline HIV (years since diagnosis) & * & $4 \cdot 6$ & $11 \cdot 3$ & $13 \cdot 4$ & 11.28 \\
\hline CD4 cell count $\left(\mathrm{x}_{10} 0^{9} / 1\right)$ & $(1.562)$ & 0.538 & $0 \cdot 252$ & $0 \cdot 313$ & $0 \cdot 313 \S$ \\
\hline HIV viral load (copies/ml) & * & 300 & 48 & 397 & \\
\hline Haemoglobin $(\mathrm{g} / \mathrm{l})$ & 78 & 72 & 55 & 39 & $63 \cdot 5$ \\
\hline Reticulocyte count (x 109/1) & 77 & 15 & 70 & 17 & $43 \cdot 5$ \\
\hline Platelet count $\left(\times \quad 10^{9} / \mathrm{l}\right)$ & 3 & 22 & 18 & 10 & 14 \\
\hline Schizocytes & + & + & + & + & \\
\hline Creatinine $(\mu \mathrm{mol} / \mathrm{l})$ & 180 & 177 & 193 & 76 & 178,5 \\
\hline $\mathrm{LDH}(\mathrm{u} / \mathrm{l})$ & 1800 & 1453 & 1600 & 2000 & 1700 \\
\hline CRP (mg/l) & 80 & 85 & 81 & 333 & 83 \\
\hline HHV8 RT-PCR (copies/ml) & 77,359 & 10,111 & $+\dagger$ & 151,189 & 77,359 \\
\hline ADAMTS13 (\%activity) & $<5 \%$ & $<5 \%$ & $<5 \%$ & $<5 \%$ & $<5 \%$ \\
\hline Anti-ADAMTS13 antibodies $(\mathrm{u} / \mathrm{ml})$ & $>100$ & 84 & 74 & 70 & 79 \\
\hline Histological examination & $\begin{array}{l}\text { Left axillary LAD } \\
\text { consistent with } \\
\text { HHV8/MCD and KS }\end{array}$ & $\begin{array}{l}\text { Infraclavicular LAD } \\
\text { consistent with } \\
\text { HHV8/MCD without KS }\end{array}$ & $\begin{array}{l}\text { Inguinal LAD consistent } \\
\text { with HHV8/MCD and } \\
\text { TMA without KS }\end{array}$ & NA & \\
\hline KS (years since diagnosis) & Yes $(2 \cdot 6)$ & Yes: & No & No & \\
\hline
\end{tabular}

TMA, thrombotic microangiopathy; MCD, Multicentric Castleman disease; HIV, Human Immunodeficiency Virus; CRP, C-Reactive Protein; HHV8, Human Herpes Virus 8; RT-PCR, Real Time Polymerase Chain Reaction; ADAMTS13, A Disintegrin And Metalloprotease with Thrombospondin type 1 motif member 13; KS, Kaposi Sarcoma; LAD, Lymphadenopathy, NA, Not available.

${ }^{*}$ Not appropriate (HIV negative).

${ }^{\dagger}$ Testing performed at another institution using qualitative PCR.

${ }^{*}$ Diagnosis was concomitant with TMA.

${ }^{\S}$ Patient 1 (HIV negative) not included. 